\title{
Metastasing presacral myxopapillary ependymoma: case report
}

\begin{abstract}
This case report focuses on a rare glial tumors with an almost exclusive localization in the distal spinal cord or in the sacrococcygeal tissue and which spread metastases only in $17 \%$ of cases - myxopapillary ependymoma. We present an unusual case pleuropulmonary dissemination of extraspinal myxopapillary ependymoma in a 28 year old female. It is described the process of differential diagnosis and definitive diagnosis on the basis of the immunohistochemical profile of the cells of the primary tumour and metastases. The diagnosis is finally determined on the basis of strong dispersive expression of GFAP (glial fibrillary acid protein), which is considered diagnostic for this type of tumor. We discuss the likely origin of the tumour as a suspected local recurrence of a sacrococcygeal tumour which was histologically probably misidentified as siringoadenoma in childhood. The discussion summarizes the available information on this low abundance neoplastic disease from the first mention of it in literature on a diagnosed tumour of cauda equina 1956 to the latest findings and the new Classification of Tumours of the Central Nervous System (2007WHO Classification of Tumours of the Central Nervous System) with subsequent application to the case of the patient.
\end{abstract}

Keywords: ependymoma, myxopapillary, extraspinal, presacral localization, dissemination
Volume 3 Issue I - 2015

\author{
Mrazkova Hana, Snajdauf Martin, Lischke \\ Robert \\ Department of Surgery, University Hospital Motol, Czech \\ Republic
}

Correspondence: Hana Mrazkova, Department of Surgery, University Hospital Motol,V Uvalu 84, 15006 Prague, Czech Republic, Tel + 420224438 00I, Fax + 420224 43I 005, Email hana.mrazkova@fnmotol.cz

Received: July 22, 2015 | Published: October 12, 2015
Abbreviations: GFAP, glial fibrillary acid protein; WHO, world health organization; CT, computer tomography; VATS, videothoracoscopic; GFAP, glial fibrillary acid protein

\section{Introduction}

Myxopapillary ependymoma is a rare tumour of the neuroepithelial tissue of glial cells. This glial neoplasm has typical localization in the distal spinal cord or in pre-/postsacral localization. Historically, it has been considered to be a benign tumour characterized by slow growth and a low likelihood of tumour dissemination..$^{1-5}$ This paper reports a rare case of extraneural presacral myxopapillary ependymoma with multiple pleuropulmonary metastases. We present the importance of early diagnosis and correct determination of the danger of gradual growth of this benign but metastatic neoplasm.

\section{Clinical history}

The 28year old female patient came to our clinic in October 2013 under the recommendation of her medical practitioner. She described pelvic pressure pain which had persevered for 2 years and which had significantly worsened in the three weeks prior to her referral. For a period of about one month she had had solid stools and difficulty with constipation. Other difficulties have been set aside. When reviewing the patient's clinical history we know that 8years prior she was operated on for a tumour of the coccyx, which according to records was histologically evaluated as papillary syringoadenoma. Given the benign nature of the finding no further action was undertaken. The patient was post-operatively monitored for the last two years for pelvic pain in a private orthopaedic clinic where she was sent for rehabilitation. Other diseases the patient negates. As an adult she underwent cosmetic surgery in 2009 for cosmetic breast implants and in April 2013 for liposuction of the thighs and abdomen.
The patient subsequently presented themselves for examination at the gynaecological clinic in September 2013. According to the findings the patient could not be examined due to rigid resistance to a suspected oppressive uterus. MR images of the abdomen and small pelvis revealed a large tumorous expansion surrounding the os sacrum with maximum pre-sacral masses, but with no signs of penetration into the bone. The tumour was putting pressure on the organs of the small pelvis. Clinically, the patient showed no visible signs of malignancy. The abdomen was not tender, there was no palpable mass, as well as no signs of peristalsis obstacles. On palpable examination of the rectum it was found rigid with immobile tumorous expansion and oppressive. During the subsequent hospitalization the only clinical symptom was pelvic pain which was managed in conjunction with the clinic for chronical pain. The patient was cardiopulmonary compensated, breathing without difficulty, auscultation normal and an oxygen saturation of $98-100 \%$.

On the basis of all the available information the patient was examined and assessed by a multidisciplinary team (oncologistsurgeon-internist-radiodiagnosticist) during the acute hospitalization. The following were performed:

i. Static Computer Tomography (CT) scans of the chest, abdomen and small pelvis with CT biopsy sampling of the sacral expansion (Figure 1). In addition to the sacral tumorous expansion the CT scan captured suspected multiple diffuse metastatic diseases of the lungs and both pulmonary hilum (Figure 2). Other organs or the skeleton were not affected.

ii. For the pulmonary findings the patient was referred for diagnostic videothoracoscopic (VATS) surgery during which stapled metastasectomy of the lingula was performed.

iii. Llaboratory tests without pathology including negative oncomarkers 

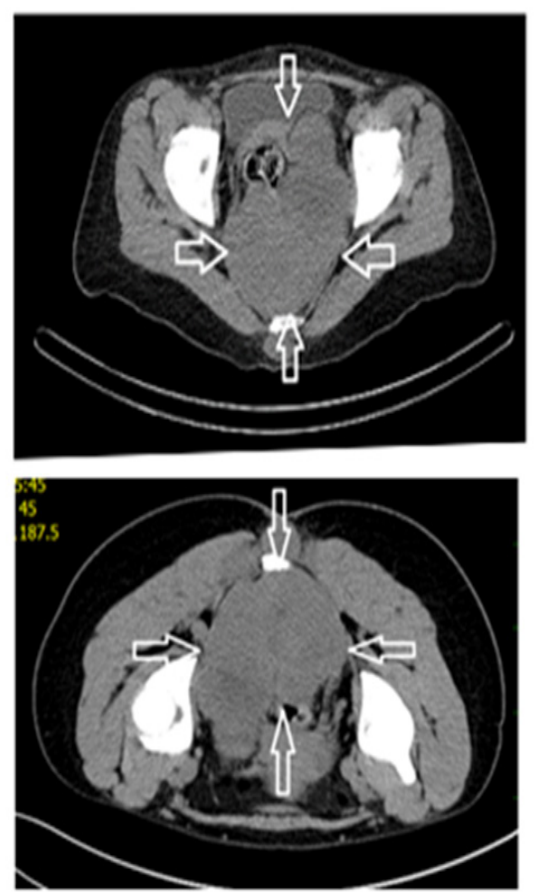

Figure I Computer tomography (CT) scan of the small pelvis - sacral expansion.

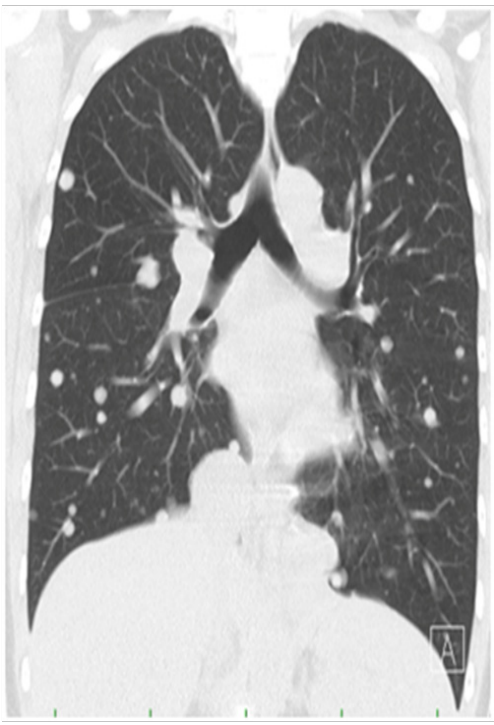

Figure 2 Computer Tomography scan of the multiple metastasizing processes.

\section{Results}

The tissue samples collected of the tumorous expansion of the small pelvis and pulmonary metastases were subjected to several weeks of histopathological analysis. The final diagnosis was that the "finding in the presacral localization is due to immunoprofile GFAP and S100 positive, which is responsible for myxopapillary ependymomas. The tumour foci in the lung are not completely of the same morphology, but the immunoprofile is similar. Comparisons with the original childhood disease are not possible because the samples have been destroyed." Given the extent of the lung disease the multidisciplinary team referred the patient for oncological care with the diagnosis myxopapillary ependymoma and multiple metastatic to the lungs.
At the time this case report went to publication the patient had undergone 3cycles of chemotherapy with CDDP, Vepesid Zarzio and 3 cycles of Doxorubicin+Neulasta and 1 palliative cycle of Vinblastin+Celocoxib. According to PET CT and MR scans the tumorous expansion of the small pelvis was without substantial change, there were multiple viable neoplasia deposits in both lungs, and newly suspected metastatic involvement in the small pelvis and rectum infiltration. The patient bears the consequences of chemotherapy- ototoxicity and cytotoxicity. Radiotherapy was considered as an additional alternative therapy for the small pelvis. After consultations with international cancer centres, and after discussions with the patient, therapy was discontinued in August 2014. The patient continued to receive symptomatic palliative therapy.

\section{Discussion}

\section{Review of literature on myxopapillary ependymoma}

Myxopapillary ependymoma is a rare glial tumour with almost exclusive localization in the distal spinal cord (intraspinal variant) or rarely in the pre- or postsacral region (extraspinal variant). ${ }^{1,2}$ The average age of manifestation has been reported to be approximately 36years. The overall incidence is approximately 0.08 per 100,000 persons per year for males and 0.05 per 100,000 persons per year for females. ${ }^{2}$ The fourth edition of the World Health Organization (WHO) Classification of Tumours of the Central Nervous System, published in 2007, states that myxopapillary ependymoma typically lacks histopathological signs of malignancy and corresponds to WHO Grade 1. This means that these tumours apply to lesions with low proliferative potential and can only be cured following surgical resection. ${ }^{1}$ A survey of literature reveals only a few reports of collected cases of different kinds of extraneural metastasizing CNS tumours. The greatest collection from Hoffman et al., ${ }^{4}$ included 282 patients of which nearly $60 \%$ were adults and $40 \%$ were children. In the adults the most common metastasizing tumour was the glioblastoma followed by meningeal tumours and medulloblastomas. Ependymomas made up only $5 \%$ of the metastasizing tumours in the age group. ${ }^{3,4}$ Distant metastasis of myxopapillary ependymoma is rare and has been suggested to occur more frequently in extraspinal than in intraspinal cases. The metastatic potential of myxopapillary ependymoma is surprising given the characteristic benign histopathology. ${ }^{2}$ Only six cases of metastases were reported in literature before $1996 .{ }^{5}$

Histologically, myxopapillary ependymoma generally contains a papillary configuration covered by cuboidal or short columnar cells. The perivascular stroma shows a characteristic mucoid or myxomatous degeneration. ${ }^{6}$ Immunohistochemically it is shown to be diagnostically a strong and disperse expression of glial fibrillary acid protein (GFAP). Less than 100cases of extradural myxopapillary epenydmoma have been described. The origin of these forms of ectopic tumour is not completely clear. ${ }^{7}$ This unusual presentation occurs in four general situations: 1) from metastases or direct extension of a primary tumour of the CNS, seen after surgical excision; 2) from direct extension to the soft tissue of the sacrococcygeal area from a primary ependymoma of the lower spinal cord, cauda equina, or filum terminale; 3) from a primary presacral, pelvic, or abdominal tumour; and 4) from a primary tumour of the skin and subcutaneous tissue of the sacrococcygeal area without demonstrable connection to the spinal cord or filum terminale. ${ }^{6-8}$ The differential diagnosis is with chordoma, extraskeletal myxoid chondrosarcoma and metastatic carcinoma, all of which can usually be readily excluded by the consistent GFAP positivity in myxopapillary ependymoma. ${ }^{9}$ 


\section{Treatment}

The therapeutic options for treating metastasizing tumours of the nervous system are very restricted. The aim is to take a gentle approach to the tumour and complete a resection during the first operation. In all cases the primary tumour responds well to radiotherapy. Intravenous systemic chemotherapy appears to be of limited benefit. ${ }^{3,10,11}$ The effectiveness of surgery and radiation therapy means that chemotherapy has been used rarely in young children with ependymomas or for the treatment of tumours that are refractory to radiation therapy ${ }^{5}$ or for the patients with distant metastasis.

\section{Conclusion-discussion of the case report}

The case presented was of presecral myxopapillary ependymoma with pulmonary tumour dissemination. From the available information we have assumed an incorrect diagnosis in childhood when the patient was operated on for a suspected tumour os sacrum with a final diagnosis of papillary siringoadenoma. Since finding myxopapillary ependymoma in the same localization, with multiple dissemination to the lungs, the conclusion is that this is likely to be a recurrence. Histological findings were confirmed by immunohistochemical analyses which revealed a strong expression of GFAP in virtually all tumour cells from biopsies taken from the presacral tumour mass and the lung metastases. Comparisons with the original histology from childhood were not possible because the samples were destroyed after 5 years in accordance with statutory guidelines. The origin of this form of pelvic myxopapillary ependymoma is probably a primary presacral, pelvic tumour without demonstrable connection to the spinal cord or filum terminale..$^{6-8}$ Due to the widespread pleurapulmonary tumour manifestations, we decided to initiate an experimental protocol of chemotherapy. Unfortunately this did not show a high rate of efficacy in halting tumour growth. Early detection of the metastases may have allowed more effective therapy like surgical metastasectomy or radiotherapy. ${ }^{2}$ Radiotherapy of the pelvic area is now being considered as palliative therapy in order to slow growth and prevent infiltration of the pelvic organs.

\section{Acknowledgements}

Supported by the project (Ministry of Health of the Czech
Republic) for the conceptual development of research organization 00064203 (University Hospital Motol, Prague, Czech Republic).

\section{Conflict of interest}

The author declares no conflict of interest.

\section{References}

1. Louis DN, Ohgaki O, Wiestler OD, et al. The 2007 WHO classification of tumours of the central nervous system. Acta Neuropathol. 2007;114(2):97-109.

2. Ilhan A, Furtner J, Birner P, et al. Myxopapillary ependymoma with pleuroplumonary metastases and high plasma glial acid protein levels. $J$ Clin Oncol. 2011;29(30):e756-e757.

3. Graf M, Blaeker H, Otto HF. Extraneural metastazing ependymoma of spinal cord. Pathology Oncology Research. 1999;5(1):56-60.

4. Hoffman HJ, Duffner PK. Extraneural metastases of central nervous system tumours. Cancer. 1985;56(7 Suppl):1778-1782.

5. Fassett DR, Pingree J, Kestle JR. The high incidence of tumour dissemination in myxopapilary ependymoma in paediatric patients. $J$ Neurosurg. 2005;102(1 Suppl):59-64.

6. Akpolat N, Bozlak N, Kazez A, et al. Sacrococcygeal extraspinal ependymoma: a case report. The Turkish Journal of Paediatrics. $2003 ; 45: 276-279$

7. Ryška A. Etraspinal Myxopapillary Ependymoma of the sacrococcygeal region. Klinická Onkologie. 2005;18(3):102-104.

8. Chung Y, Lee SK, Yang KH, et al. Subcutaneous sacrococcygeal myxopapillary ependymoma. Med Pediatr Oncol. 1999;30(2):81-84.

9. Graadt van Roggen JF, Hogendoorn PCW, Fletcher CD. Myxoid tumours of soft tissue. Histopathology. 1999;35(4):291-312.

10. Morris DM, Steinert HR, Wiernik PH. Ineffectiveness of chemotherapy in patients with metastatic ependymoma of the cauda equina. J Surg Oncol. 1983;22(1):33-36.

11. Sgouros S, Malluci CL, Jackowski A. Spinal ependymomas-the value of postoperative radiotherapy for residual disease control. Br J Neurosurg. 1996;10(6):559-566. 